\title{
Análise do comportamento cardiorrespiratório em indivíduos com doença pulmonar obstrutiva diante do treino aeróbico por meio da caminhada nórdica
}

\author{
Analysis cardiorrespiratory in individuals with obstructive \\ pulmonary disease in aerobic training with nordic walking
}

Gabriela Quaresma da Rocha', Aline Silva Castro', Emilly Luzia dos Santos Sousa', Leonardo de Oliveira Chaves ${ }^{1}$, Luiz Fabio Magno Falcão ${ }^{2}$, Valéria Marques Ferreira Normando ${ }^{3}$

\begin{abstract}
Resumo Objetivo: Analisar o comportamento cardiorrespiratório de indivíduos com doença pulmonar obstrutiva diante do treinamento aeróbico por meio da caminhada nórdica. Método: Trata-se de um estudo longitudinal, prospectivo, quantitativo, de centro único. Realizado com oito voluntários de 51 a 84 anos, que foram distribuídos em G1- Caminhada nórdica (n=5) e G2- Caminhada tradicional ( $\mathrm{n}=3$ ). Foram submetidos ao teste de caminhada de 6 minutos (TC6), sendo utilizados bastões nórdicos para o G1 e caminhada livre para o G2. Resultados: Observou-se diferença estatística em relação ao aumento da pressão arterial sistólica final do grupo do $\mathrm{G} 2(\mathrm{p}=0,000)$. Na comparação entre os grupos independentes, a frequência cardíaca inicial obteve resultado significativo ( $\mathrm{p}=0,003)$, assim como na medida da saturação periférica de oxigênio antes e após o treino aeróbico nos dois grupos ( $\mathrm{p}=0,000)$ e nos valores finais da escala de BORG, no qual o G1 obteve média menor ( $\mathrm{p}=0,02)$. Conclusão: Concluiu-se que o treino aeróbico por meio da caminhada nórdica pode ter contribuído para a manutenção da pressão arterial sistólica e frequência cardíaca inicial dos indivíduos com doença pulmonar obstrutiva, assim como para a diminuição do valor da percepção de esforço pela escala de BORG.
\end{abstract}

Descritores: caminhada; pneumopatias obstrutivas; exercício, fisioterapia.

Summary Purpose: To analyze the cardiorespiratory behavior of individuals with obstructive pulmonary disease before aerobic training through Nordic walking. Method: It is a longitudinal, prospective, quantitative, single center study. It was carried out with eight volunteers aged 51 to 84 years, who were distributed in G1 - Nordic walking (n=5) and G2 - Traditional walking $(n=3)$. They were submitted to the 6-minute walk test (6MWT), using Nordic walking sticks for G1 and walking free for G2. Results: Statistical difference was observed in relation to the increase in the final systolic blood pressure of the $G 2$ group ( $p=0.000$ ). In the comparison between the independent groups, the initial heart rate obtained a significant result ( $p=0.003)$, as well as in the measurement of peripheral oxygen saturation before and after aerobic training in both groups $(\mathrm{p}=0.000)$ and in the final values of the BORG, in which the G1 obtained a lower mean $(\mathrm{p}=0.02)$. Conclusion: It was concluded that aerobic training through Nordic walking may have contributed to the maintenance of systolic blood pressure and initial heart rate in individuals with obstructive pulmonary disease, as well as to decrease the value of perceived exertion by the BORG scale.

Keywords: walking; lung diseases; obstructive; exercise; physiotherapy.

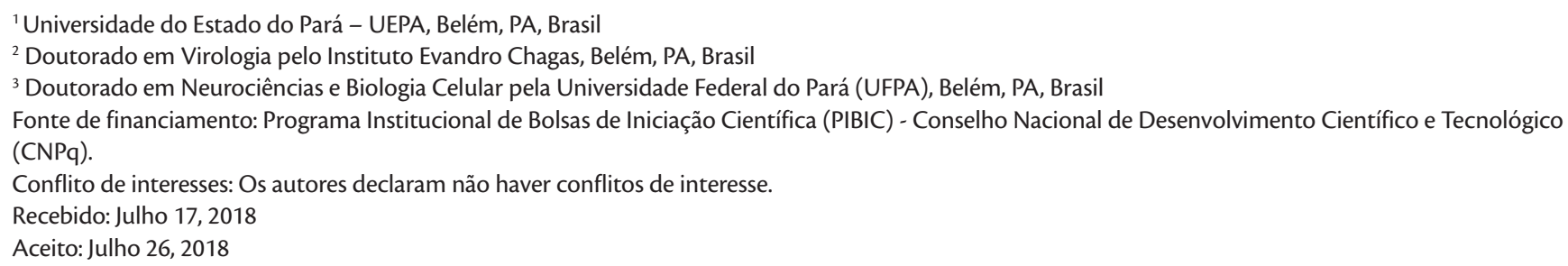

Trabalho realizado no Centro de Ciências Biológicas e da Saúde Campus II da Universidade do Estado do Pará - UEPA, Belém, PA, Brasil. 


\section{Introdução}

O sistema mucociliar precisa manter a sua estrutura, sincronia, quantidade e qualidade de secreção brônquica em perfeita interação para um bom funcionamento ${ }^{1}$. A doença pulmonar obstrutiva é caracterizada por afetar a integridade do sistema mucociliar, onde ocorre um aumento da resistência ao fluxo expiratório pelo estreitamento das vias aéreas, podendo ser considerado leve, moderado e grave ${ }^{2}$. Os distúrbios obstrutivos mais conhecidos são a asma, a doença pulmonar obstrutiva crônica (DPOC), fibrose cística e bronquiectasia ${ }^{2,3}$. A progressão da doença leva a piora da dispneia, perda de força muscular periférica e limitação na realização das atividades de vida diária³

A DPOC é um fator de risco associado à morbimortalidade por doenças cardiovasculares. Em indivíduos com doença obstrutiva a rigidez arterial tem se mostrado elevada. Apesar de não se ter resultados coerentes quanto à relação desta rigidez com a limitação do fluxo aéreo e inflamação sistêmica sabe-se que ela aumenta o risco para aterosclerose possibilitando uma maior chance de ocorrerem episódios cardiovasculares ${ }^{4}$.

A fraqueza muscular periférica é um dos principais fatores de diminuição da capacidade funcional na doença obstrutiva, pois a endurance muscular diminuída reflete no aumento da fadiga ${ }^{5}$. A reabilitação pulmonar com ênfase no treinamento físico tem mostrado eficácia na tolerância ao esforço, aumentando a capacidade de realização das atividades de vida diária, tendo em vista que o exercício é recomendado para todo indivíduo com doença pulmonar obstrutiva ${ }^{6,7}$. A capacidade física é o modo como as reações fisiológicas que ocorrem no indivíduo se expressam, logo, a reabilitação pulmonar busca melhorar esta capacidade por meio de exercícios aeróbicos ${ }^{8,9}$.

Atualmente, o exercício aeróbico é considerado uma intervenção essencial na reabilitação cardiorrespiratória ${ }^{10}$. Através de exercícios aeróbicos é possível observar melhorias no transporte mucociliar de indivíduos com doença obstrutiva, sugerindo uma melhor adaptação e eficiência do sistema cardiorrespiratório ${ }^{11}$. A literatura chama a atenção para a condição relevante da reabilitação, o qual se utiliza do treinamento físico, na redução dos sintomas prevalentes como dispnéia e fadiga e, consequentemente, à melhora da capacidade de realizar os exercícios ${ }^{3}$.

Como componente da reabilitação cardiorrespiratória, a caminhada tradicional vem ser destaque no meio da prática de exercícios, por ser de fácil execução, baixo custo e baixo risco a saúde. Este é um exercício físico frequentemente utilizado para indivíduos com doenças cardíacas e pulmonares ${ }^{7}$. Recentemente, para somar na reabilitação cardiopulmonar, a literatura ressalta a Caminhada Nórdica como recurso complementar. Esta começou a ser realizada na Europa como forma de tratamento para pacientes portadores de enfermidades do sistema cardiorrespiratório ${ }^{12}$.

A Caminhada Nórdica é um tipo de caminhada em que se utilizam dois polos concebidos especialmente para este exercício, que auxiliam nas fases da marcha, onde o indivíduo empurra cada polo em um ângulo diagonal criando força motriz para um estilo de andar mais ativo. Apesar de poucos relatos sobre a cinemática desta caminhada, ela tem sido cada vez mais utilizada como terapia física ${ }^{13}$. A principal característica que a difere da caminhada tradicional é ser possível realizá-la sem aumentar a percepção de esforço, pois reduz o impacto do peso corporal sobre os membros inferiores e ao mesmo tempo provoca movimentação nos membros superiores e tronco, possibilitando um aumento da capacidade aeróbia ${ }^{14}$.

Cerca de $90 \%$ dos músculos do corpo são utilizados na caminhada nórdica, entre seus benefícios está o reforço muscular das extremidades superiores do corpo, aumento da mobilidade do segmento superior da coluna, alívio do estresse sobre as articulações, correção de posturas viciosas entre outros. O consumo de calorias é de $67 \%$ a mais na caminhada nórdica quando comparada a corrida ${ }^{15}$.

Tendo em vista os benefícios até então citados na literatura sobre a caminhada nórdica em reabilitação cardiopulmonar, o objetivo deste estudo foi analisar o comportamento cardiorrespiratório de indivíduos com doença pulmonar obstrutiva diante o treinamento aeróbico por meio da caminhada nórdica.

\section{Metodologia}

Trata-se de um estudo longitudinal, prospectivo, quantitativo, de centro único, realizado no Centro de Ciências Biológicas e da Saúde (CCBS) da Universidade do Estado do Pará (UEPA) entre agosto de 2015 a junho de 2016, sob parecer de aprovação $n^{\circ} 1.117 .410$ do Comitê de Ética e Pesquisa da Universidade do Estado do Pará (UEPA). 
Oito voluntários com idade entre 51 a 84 anos, do sexo masculino $(n=5)$ e feminino $(n=3)$, ex tabagistas, aposentados, residentes de Belém - PA, oriundos dos Sistema Único de Saúde (SUS) e procedentes do Ambulatório de Fisioterapia Respiratória do Centro Especializado em Reabilitação II (CER II) aceitaram participar do estudo assinando o Termo de Consentimento Livre e Esclarecido (TCLE). Todos com diagnóstico de Doença Pulmonar Obstrutiva grau leve ou moderado segundo o II Consenso Brasileiro sobre Doença Pulmonar Obstrutiva Crônica. Do total da amostra previamente selecionada foram excluídos aqueles indivíduos que apresentaram alguma doença associada como artrose $(n=2)$ e osteoporose $(n=1)$, que não conseguiram terminar o teste de caminhada de 6 minutos $(n=1)$, bem como aqueles que se ausentaram por duas vezes durante o período de realização das sessões de treino aeróbico $(n=1)$.

Os voluntários foram divididos aleatoriamente em dois grupos: G1: Caminhada nórdica $(n=5)$ e G2: Caminhada tradicional $(n=3)$. Foram submetidos ao treino aeróbico por meio do teste de caminhada de 6 minutos em uma área plana com 30 metros sob incentivo verbal da avaliadora, sendo que o G1 utilizou bastões nórdicos durante as caminhadas e o G2 conduziu a caminhada livre, sem auxílio.

Durante o treinamento foram monitorados a saturação periférica de oxigênio $\left(\mathrm{SpO}_{2}\right)$ pré e pós teste por meio de oxímetro de pulso, juntamente com a percepção de dispnéia segundo a escala de Borg modificada. A distância percorrida foi quantificada em metros. Antes e depois do teste foram aferidos o pulso periférico e a pressão arterial.

As informações coletadas foram analisadas por meio do software Minitab ${ }^{\circledR} 14$, Minitab ${ }^{\circledR}($ Minitab, Inc., in the United State). Foram utilizados o teste $t$ de Student e teste t pareado na análise estatística, adotando-se nível $\alpha$ de 0.05 para rejeição da hipótese nula.

\section{Resultados}

Dos indivíduos que participaram do estudo 62,5\% (5/8) eram do gênero masculino e 37,5\% (3/8) do gênero feminino, média de idade $66,75 \pm 10,97$ anos, realizavam o protocolo de reabilitação pulmonar há \pm 4 anos e eram portadores de doença pulmonar obstrutiva, sendo o grupo G1: enfisema pulmonar e bronquite crônica $(n=4)$, bronquiectasia $(n=1)$ e o grupo $G 2$ : asma $(n=1)$ e bronquiectasia $(n=2)$. A Tabela 1 mostra os demais dados antropométricos.

A Tabela 2 mostra a comparação entre as médias iniciais e finais da Pressão Arterial Sistólica e Pressão Arterial Diastólica dos dois grupos. Observou-se diferença estatisticamente significante apenas na Pressão Arterial Sistólica no G2, no qual houve um aumento $(\mathrm{p}=0,000)$.

Os resultados mostraram uma variação da FC estatisticamente significante entre os valores iniciais e finais de ambos os grupos $(p=0,000)$. Entretanto, quando realizada a comparação entre os grupos independentes, apenas os valores de FC iniciais apresentaram alteração significativa $(\mathrm{p}=0,003)$. A média dos valores da FC inicial do G1 foi estabelecida em $80,05 \pm 10,22$, em contrapartida o G2 obteve média 88,88 $\pm 21,15$, como mostra o Figura 1.

Houve diferença estatisticamente significante na medida da $\mathrm{SpO}_{2}$ antes e após o treino nos dois grupos $(\mathrm{p}=0,000)$. Como ilustrado no Figura 2, as médias do grupo de caminhada nórdica variaram de 95,35 no início para 91,41 ao final. Já os valores médios do grupo de caminhada tradicional foram de 95,75 e 92,68,

Tabela 1. Dados antropométricos dos indivíduos, UEPA, 2016

\begin{tabular}{ccc}
\hline Variáveis & $\begin{array}{c}\text { G1 } \\
\text { Caminhada Nórdica } \\
(\mathbf{n}=5)\end{array}$ & $\begin{array}{c}\text { G2 } \\
\text { Caminhada Tradicional } \\
(\mathbf{n}=3)\end{array}$ \\
Idade $^{1}$ & 63,2 anos $^{1}$ & 72,6 anos $^{1}$ \\
& $\pm 10,13$ & $\pm 11,5$ \\
Altura $^{1}$ & $1,58 \mathrm{~m}^{1}$ & $1,54 \mathrm{~m}^{1}$ \\
& $\pm 0,06$ & $\pm 0,04$ \\
\hline Peso $^{1}$ & $61,8 \mathrm{~kg}^{1}$ & $62,6 \mathrm{~kg}^{1}$ \\
& $\pm 14,09$ & $\pm 6,8$ \\
IMC $^{1}$ & $24,6^{1}$ & $26,15^{1}$ \\
& $\pm 5,1$ & $\pm 1,85$
\end{tabular}

IMC: índice de massa corpórea. ${ }^{1}$ valores obtidos por cálculo da média. Fonte: Autor, 2016. 
Tabela 2. Comparação entre médias iniciais e finais da Pressão Arterial, UEPA, 2016

\begin{tabular}{ccc}
\hline Variáveis & $\begin{array}{c}\text { G1 } \\
\text { Caminhada Nórdica }(\mathbf{n = 5})\end{array}$ & $\begin{array}{c}\text { G2 } \\
\text { Caminhada Tradicional }(\mathbf{n}=\mathbf{3})\end{array}$ \\
\hline PAS inicial & $122,92 \pm 11,03$ & $124,80 \pm 12,13$ \\
\hline PAS final & $124,41 \pm 12,16$ & $133,43 \pm 13,78$ \\
\hline Valor $p$ & $\mathbf{0 , 2 4 7}$ & $\mathbf{0 , 0 0 0}$ \\
\hline PAD inicial & $72,80 \pm 8,30$ & $72,27 \pm 10,44$ \\
\hline PAD final & $73,40 \pm 10,47$ & $73,60 \pm 13,75$ \\
\hline Valor $p$ & $\mathbf{0 , 5 6 6}$ & $\mathbf{0 , 4 1 5}$ \\
\hline
\end{tabular}

PAS: pressão arterial sistólica; PAD: pressão arterial diastólica. Fonte: Autor, 2016.

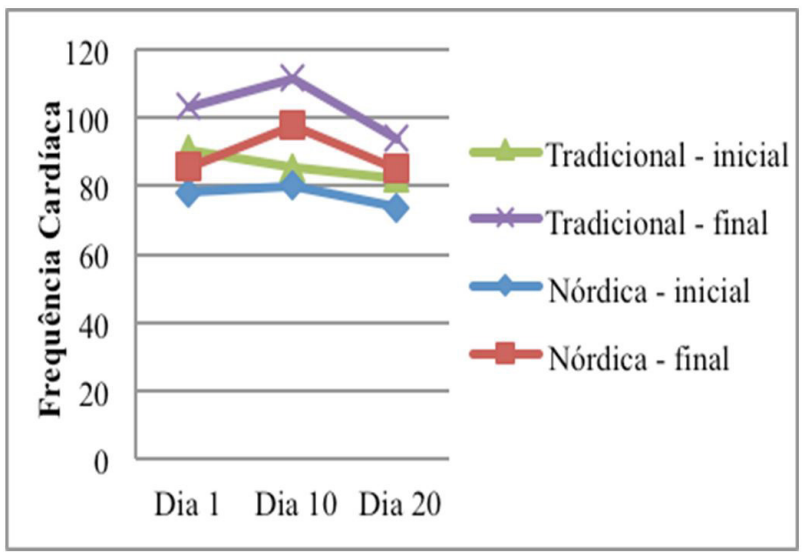

Figura 1. Médias da frequência cardíaca inicial e final no $1^{\circ}, 10^{\circ}$ e $20^{\circ}$ dias.

Fonte: Autor, 2016.

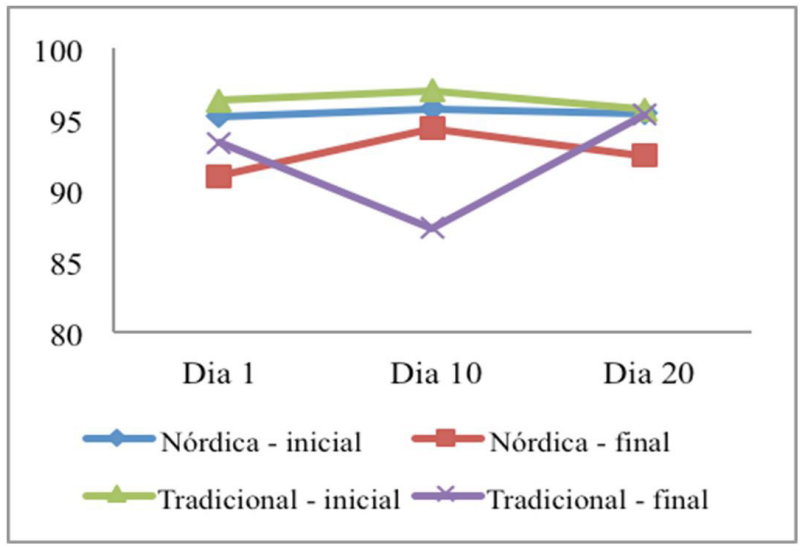

Figura 2. Médias da saturação periférica inicial e final no $1^{\circ}, 10^{\circ}$ e $20^{\circ}$ dias.

Fonte: Autor, 2016

respectivamente. Porém, apesar das alterações clínicas, não se observou significância estatística quando comparados os dois grupos independentes.

No grupo de caminhada tradicional a distância percorrida foi menor obtendo média inferior ao grupo de caminhada nórdica, porém, não houve resultado estatisticamente significante como se pode observar na Tabela 3.

Os dois grupos atingiram média maior de percepção de esforço ao final das caminhadas, resultando em uma diferença estatisticamente significativa quando comparado o pré-teste e o pós-teste de cada grupo. $\mathrm{Na}$ análise entre os grupos apenas os valores de percepção do esforço do pós-treino apresentou resultado significativo, onde a média do G2 foi maior que G1, como mostra a Tabela 3. 
de 3,07. Apesar da diferença discreta entre a queda de saturação dos dois grupos, os resultados confirmam o que foi dito na literatura, onde a dessaturação é maior na caminhada nórdica.

As distâncias percorridas não apresentaram resultados significantes estatisticamente como no estudo de Takeshima et al. ${ }^{25}$, o qual revelou que o grupo de caminhada nórdica percorreu uma menor distância que o grupo de caminhada tradicional, porém, não houve resultado significativo também. A média percorrida pelo grupo nórdico foi maior neste estudo, porém, acredita-se que a diferença do número de participantes entre os grupos tenha influenciado no resultado.

A doença pulmonar obstrutiva diminui a capacidade de exercício, logo, é possível observar que todo teste submáximo provoca alterações fisiológicas importantes nestes indivíduos, porém, alguns provocam maior alteração que outros. Em um estudo de indivíduos com DPOC que praticaram TC6, a média da escala de percepção de esforço de BORG foi 3,6 6 2,5, o que é considerado pouco forte ${ }^{26}$. A caminhada nórdica provoca maior movimentação corporal que outros tipos de caminhada, devido à utilização dos membros superiores e tronco para mover os bastões ${ }^{21}$. Apesar do maior consumo de oxigênio durante a caminhada nórdica, a percepção de esforço parece estar diminuída, melhorando a pontuação da escala de BORG ${ }^{27}$. A pontuação da escala de BORG obteve aumento nos dois tipos de caminhada deste estudo, porém, analisando os dois grupos, houve resultado significativo apenas no pós-treino, onde o G1 conseguiu média menor que G2.

Uma limitação do estudo foi o número reduzido da amostra, a qual pode ter influenciado alguns resultados, logo, um estudo com maior população poderia indicar maior exatidão dos parâmetros pesquisados.

\section{Conclusão}

Os resultados sugerem que a caminhada tradicional, diferentemente da caminhada nórdica, promove um aumento da pressão arterial sistólica. Os dois modos de caminhada provocam a diminuição da $\mathrm{SpO}_{2^{\prime}}$ aumento da FC e do valor de BORG. Entretanto, quando comparados os valores iniciais ao treino dos dois grupos, a caminhada nórdica apresentou uma média menor de FC e no pós-treino os valores de BORG foram menores no grupo de caminhada nórdica.

Esta pesquisa ressalta a importância de novos estudos acerca da caminhada nórdica e seus benefícios na reabilitação cardiorrespiratória para que haja uma melhor definição dos resultados.

\section{Referências}

1. Leite MR, Ramos EMC, Freire APCF, Silva BSA, Nicolino J, Toledo AC et al. Efeito agudo do exercício aeróbio em diferentes intensidades no transporte mucociliar de pacientes com DPOC. Medicina. 2015;48(6):523-32.

2. Minatel V, Saad IAB, Moraes M, Córdova Jr VA, Saad BA, Quagliato Jr R. Avaliação da distância percorrida e velocidade média durante o TC6 em pacientes com diferentes doenças pulmonares. J Health Sci Inst. 2012;30(3):281-6.

3. Araújo CLP, Karloh M, Santos KD, Reis CM, Mayer AF. Reabilitação pulmonar em longo prazo na doença pulmonar obstrutiva crônica. ABCS Health Sciences. 2014;39(1):56-60. http://dx.doi.org/10.7322/abcshs.v39i1.246.

4. Sievi NA, Franzen D, Kohler M, Clarenbach CF. Physical inactivity and arterial stiffness in COPD. Int J Chron Obstruct Pulmon Dis. 2015;10:1891-7. PMid:26392763.

5. Pedrozo MD. O equilíbrio e o medo de sofrer quedas em pacientes portadores da doença pulmonar obstrutiva crônica [dissertação]. Santa Maria (RS): Programa de Pós-graduação de Distúrbios da Comunicação Humana, Universidade Federal de Santa Maria - UFSM; 2012.

6. Costa CC, Leite BS, Canterle DB, Souza RM, Machado ML, Teixeira PJZ. Análise da força, qualidade de vida e tolerância ao exercício na doença pulmonar crônica. R Bras Ci e Mov. 2014;22(2):27-35. http://dx.doi.org/10.18511/0103-1716/rbcm.v22n2p27-35.

7. Queiroz ACC, Brito LC, Santos MA, Fecchio RY, Stocco ALB, Bezerra Al, et al. Prescrição de caminhada não supervisionada, risco cardiovascular e aptidão física. Rev Bras Educ Fís Esporte. 2013 Jul-Set;27(3):377-86.

8. Ambrozin ARP, Paschoal AMJ, Raquel DFS, Borges JB, Quitério RJ. Associação do treinamento resistido e aeróbico em pacientes com doença pulmonar crônica. Rev Ter Man. 2013;11(53):327-32.

9. Moraes EZC, Trevisan ME, Baldisserotto SV, Portela LOC. Capacidade aeróbica em crianças e adolescentes com asma intermitente e persistente leve no período intercrises. J Bras Pneumol. 2012;38(4):438-44. http://dx.doi.org/10.1590/S1806-37132012000400005.

10. Rocha Leite M, Kalva-Filho CA, Coelho Figueira Freire AP, Spolador de Alencar Silva B, Nicolino J, Toledo-Arruda A, et al. Effects of 12 weeks of aerobic training on autonomic modulation, mucociliary clearance, and aerobic parameters in patients with COPD. Int J Chron Obstruct Pulmon Dis. 2015;10:2549-57. http://dx.doi.org/10.2147/COPD.S81363. PMid:26648712.

11. Pessoa BV, Jamami M, Basso RP, Regueiro EMG, Di Lorenzo VAP, Costa D. Teste do degrau e teste da cadeira: comportamento das respostas metábolo-ventilatórias e cardiovasculares na DPOC. Fisioter Mov Curitiba. 2012 jan/mar;25(1):105-15. http://dx.doi.org/10.1590/ S0103-51502012000100011. 
12. Shim JM, Kwon HY, Kim HR, Kim BI, Jung JH. Comparison of the effects of walking with and without nordic pole on upper extremity and lower extremity muscle activation. J Phys Ther Sci. 2013;25(12):1553-6. http://dx.doi.org/10.1589/jpts.25.1553. PMid:24409018.

13. Homma D, Jigami H, Sato N. Effects of Nordic walking on pelvis motion and muscle activities around the hip joints of adults with hip osteoarthritis. J Phys Ther Sci. 2016 abr;28(4):1213-8. http://dx.doi.org/10.1589/jpts.28.1213. PMid:27190455.

14. Arbillaga-Etxarri A, Torrent-Pallicer J, Gimeno-Santos E, Barberan-Garcia A, Delgado A Balcells E, et al. Validation of walking trails for the urban training TM of chronic obstructive pulmonary disease patients. PLoS One. 2016;11(1):1-11. http://dx.doi.org/10.1371/journal. pone.0146705. PMid:26766184.

15. Hagner-Derengowska M, Kałużny K, Hagner W, Kochański B, Plaskiewicz A, Borkowska A, et al. The influence of a ten-week Nordic walking training-rehabilitation program on the level of lipids in blood in overweight and obese postmenopausal women. J Phys Ther Sci. 2015 Out;27(10):3039-44. http://dx.doi.org/10.1589/jpts.27.3039. PMid:26644639.

16. Portillo K, Torralba Y, Blanco I, Burgos F, Rodriguez-Roisin R, Rios J, et al. Pulmonary hemodynamic profile in chronic obstructive pulmonary disease. Int J Chron Obstruct Pulmon Dis. 2015;10:1313-20. http://dx.doi.org/10.2147/COPD.S78180. PMid:26203238.

17. Kikuchi H, Shiozawa N, Takata S, Ashida K, Mitsunobu F. Effect of repeated Waon therapy on exercise tolerance and pulmonary function in patients with chronic obstructive pulmonary disease: a pilot controlled clinical trial. Int J Chron Obstruct Pulmon Dis. 2014;9:9-15. PMid:24363555.

18. Squassoni SD, Machado NC, Lapa MS, Cordoni PK, Bortolassi LC, Oliveira JN, et al. Comparison between the 6-minute walk tests performed in patients with chronic obstructive pulmonary disease at different altitudes. Einstein. 2014 Oct./Dec;12(4):447-51.

19. Andrianopoulos V, Wagers SS, Groenen MT, Vanfleteren LE, Franssen FM, Smeenk FW, et al. Characteristics and determinants of endurance cycle ergometry and six-minute walk distance in patients with COPD. BMC Pulm Med. 2014;14(97):1-10. http://dx.doi.org/10.1186/14712466-14-97. PMid:24885117.

20. Cordeiro ALL, Amorim NM, Andrade PH, Esquivel MS, Guimarães AR, Melo TA, et al. Alterações fisiológicas da caminhada e tempo de internamento no pós-operatório de cirurgia cardíaca. Int J Cardiovasc Sci. 2015;28(5):480-6.

21. Pellegrini B, Peyré-Tartaruga LA, Zoppirolli C, Bortolan L, Bacchi E, Figard-Fabre H, et al. Exploring muscle activation during nordic walking: a comparison between conventional and uphill walking. PLoS One. 2015;10(9):e0138906. http://dx.doi.org/10.1371/journal.pone.0138906. PMid:26418339.

22. Facco CR, Soares JC, Mota CB, Trevisan ME. Efeitos de um teste incremental de caminhada na marcha de indivíduos com Doença Pulmonar Obstrutiva Crônica. Fisioter Pesqui. 2014;21(1):47-52. http://dx.doi.org/10.1590/1809-2950/441210114.

23. Moreira MAF, Medeiros GA, Boeno FP, Sanches PRS, Silva Jr DP, Muller AF. Análise da dessaturação de oxigênio durante o teste de caminhada de seis minutos em pacientes com DPOC. J Bras Pneumol. 2014 Mai./Jun.;40(3):222-228.

24. Sugiyama K, Kawamura M, Tomita $\mathrm{H}$, Katamoto S. Oxygen uptake, heart rate, perceived exertion, and integrated electromyogram of the lower and upper extremities during level and Nordic walking on a treadmill. J Physiol Anthropol. 2013;32(2):1-9. http://dx.doi.org/10.1186/18806805-32-2. PMid:23406834.

25. Takeshima N, Islam MM, Rogers ME, Rogers NL, Sengoku N, Koizumi D, et al. Effects of nordic walking compared to conventional walking and band-based resistance exercise on fitness in older adults. J Sports Sci Med. 2013 Set;12(3):422-30. PMid:24149147.

26. Mazzocchi CS, Costa CC, Canterle DB, Moussalle LD, Colombo C, Teixeira PJZ. Comparação das variáveis fisiológicas no teste de caminhada de seis minutos e no teste da escada em portadores de doença pulmonar obstrutiva crônica. Rev Bras Med Esporte. 2012 Set./Oct.;18(5):296-9.

27. Breyer MK, Breyer-Kohansal R, Funk GC, Dornhofer N, Spruit MA, Wouters EF, et al. Nordic Walking improves daily physical activities in COPD: a randomised controlled trial. Respir Res. 2010;11(112):1-9. http://dx.doi.org/10.1186/1465-9921-11-112. PMid:20727209.

\section{Autor correspondente}

Gabriela Quaresma da Rocha

Passagem Bom Jardim, 37, Pedreira

CEP 66083-120, Belém, PA, Brasil

E-mail: gabrielaquaresmarocha@gmail.com

Informação sobre os autores

GQR e ELSS são Fisioterapeutas graduadas pela Universidade do Estado do Pará (UEPA)

ASC e LOC são acadêmicos do curso de graduação em Fisioterapia da Universidade do Estado do Pará (UEPA).

LFMF é doutor em virologia pelo Instituto Evandro Chagas.

VMFN é doutora em Neurociências e Biologia Celular pela Universidade Federal do Pará (UFPA).

\section{Contribuição dos autores}

GQR foi responsável pela escrita e coleta de dados; ASC, LOC e ELSS foram responsáveis pela coleta de dados; LFMF realizou a metologia estatística; VMFN foi responsável pela orientação e correção do artigo. 